\title{
Deep Anterior Lamellar Keratoplasty Using the Big-Bubble Technique in Keratoconus
}

\author{
(1) Sibel Ahmet, ${ }^{1}$ () Nilay Kandemir Besek, ${ }^{2}$ () Alper Agca, ${ }^{2}$ (i) Muhittin Taskapili² \\ 1Department of Ophthalmology, Agri State Hospital, Agri, Turkey \\ ${ }^{2}$ University of Healty Sciences, Beyoglu Eye Training and Research Hospital, Istanbul, Turkey
}

\begin{abstract}
Objectives: The aim of this study was to evaluate the visual outcomes and complications of deep anterior lamellar keratoplasty (DALK) performed using the big-bubble technique in patients with keratoconus.

Methods: In this case series, 57 eyes of 57 patients with moderate to advanced keratoconus underwent DALK. All of the participants were contact lens-intolerant or had an unacceptable contact lens fitting as a result of advanced keratoconus. DALK was performed using the big-bubble technique. Full thickness donor corneas devoid of Descemet's membrane were sutured to the recipient bed. Preoperative and postoperative best spectacle-corrected visual acuity (BSCVA), corneal topography, and intra-postoperative complications were evaluated.

Results: The mean age of 40 male $(70 \%)$ and 17 female $(30 \%)$ patients who had an average follow-up of $48.84 \pm 18.12$

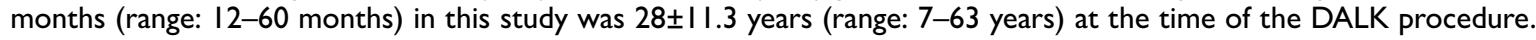
The mean BSCVA increased from I.4I $\pm 0.44 \log M A R$ to $0.87 \pm 0.37 \log M A R$ at the final follow-up $(p<0.00 I)$. The mean preoperative value of flat $(\mathrm{KI})$ and steep $(\mathrm{K} 2)$ curvature power was $53.5 \pm 8.8$ diopters $(\mathrm{D})$ and $60.6 \pm 8.5 \mathrm{D}$, respectively, while the postoperative mean KI and $\mathrm{K} 2$ value was $42.8 \pm \mathrm{I} .2 \mathrm{D}$ and $46.06 \pm \mathrm{I} .3 \mathrm{D}$. Microperforation was seen in 6 patients (10.5\%) during the suturation as an intraoperative complication. Postoperatively, 8 patients (14\%) underwent suture revision and I patient $(0.2 \%)$ underwent amniotic membrane transplantation.

Conclusion: DALK using the big-bubble technique appears to be a safe and effective procedure in patients with keratoconus. Keywords: Big-bubble, deep anterior lamellar, keratoconus, keratoplasty.
\end{abstract}

\section{Introduction}

There are 3 basic approaches to keratoconus treatment. There are methods that provide rehabilitation of the patient's vision (glasses, hard contact lenses, intracorneal segment insertion), stop the progression of the disease (crosslinking), and remove the source of the disease (keratoplasty). Surgery is required when the disease has become advanced and the patient's vision can no longer be rehabilitated with glasses or contact lenses ( 1 ).

The surgical treatment technique of keratoplasty for keratoconus can be applied using 2 methods: penetrating keratoplasty (PK) or deep anterior lamellar keratoplasty (DALK).
Though PK adversely affects the structure and integrity of the eye, it has commonly been used as the definitive treatment for many corneal pathologies, such as keratoconus and corneal degeneration or dystrophy (2).

DALK is a method that doesn't affect the endothelium or Descemet's membrane (DM), so it can be used in cases of corneal disease located above the DM (3). Although DALK is a time-consuming procedure and a method that requires experience, it has advantages, including the elimination of endothelial graft rejection, reduction of graft failure, faster visual rehabilitation, and longer graft survival due to lower rates of endothelial cell loss (4). Rather than manual dis- 
section, the injection of air or viscoelastic material into the deep corneal stroma in DALK provides for easier separation of the DM from the stroma and leaves a smoother and more uniform interface, thus reducing the likelihood of complications, such as interface opacity (5). The purpose of this study was to report and assess the results of DALK using the bigbubble technique in patients with keratoconus.

\section{Methods}

The medical records of patients who had undergone DALK with the big-bubble technique for moderate to advanced keratoconus between February 2012 and February 2017 were retrospectively reviewed. The study was conducted according to the tenets of the Declaration of Helsinki. Written informed consent was obtained from all of the patients before the DALK procedure was performed. Keratoconus was diagnosed clinically based on slit lamp findings (stromal thinning, Fleischer ring, Vogt's striae) and keratometry, and was confirmed by corneal topography. The study inclusion criteria were moderate to advanced keratoconus with poor best spectacle-corrected visual acuity (BSCVA), rigid gas-permeable contact lens intolerance, or inappropriate contact lens fit. Exclusion criteria included ocular diseases that may affect measurements or visual acuity gains, such as vernal keratoconjunctivitis (VKC), or the presence of hydrops, cataracts, retinal disorders, or glaucoma. Preoperatively, all of the patients had a full ophthalmological examination to determine uncorrected visual acuity (UCVA), BSCVA, manifest and cycloplegic refractions, slit lamp evaluation, Goldman applanation tonometry, and fundoscopy. Using the Sirius topography system (Costruzioni Strumenti Oftalmici, Florence, Italy), the following topographic parameters were recorded and evaluated preoperatively and postoperatively: the flattest keratometric reading $(\mathrm{KI})$ and the steepest keratometric reading $(\mathrm{K} 2)$. All of the patients were operated on using the big-bubble technique. The diameter of trephination was chosen according to the size of the cone and vertical corneal diameter. After trephination to approximately $80 \%$ of the corneal thickness with a Hessburg-Barron suction trephine (Katena Products Inc., Denville, NJ, USA), a 27-gauge needle attached to a 5-cc syringe and bent at $100^{\circ}$ (bevel facing downward) was inserted into the stroma up to the center of the cornea. Air was gently injected into the midstroma until a big bubble was formed extending to the border of trephination. If no big bubble was formed the first time, the injection was repeated until a big bubble was formed. After bubble formation, debulking of the anterior two-thirds of the corneal stroma was performed with a crescent blade (Alcon Laboratories, Fort Worth, TX, USA). Thereafter, peripheral paracentesis was performed to reduce intraocular pressure, and the bubble was punctured with a $15^{\circ}$ slit-knife
(Alcon Laboratories, Fort Worth, TX, USA) to allow for air to escape and collapse of the bubble. Viscoelastic material (Coatel, Bausch \& Lomb, Inc., Bridgewater, NJ, USA) was injected to keep the DM away from the manipulations. Vannas scissors were used to divide the rest of the corneal stroma into 4 quadrants and each quadrant was completely excised using left and right transplantation scissors, taking care not to leave any posterior lip. The viscoelastic material was then completely washed out before proceeding to graft suturing. If DM perforation occurred and was large enough to preclude lamellar keratoplasty, the procedure was converted to PK. Data related to these eyes were excluded from the study. The donor cornea was punched from the endothelial side using the Barron punch (Katena Products Inc., Denville, $\mathrm{NJ}$, USA). The donor material was oversized by $0.5 \mathrm{~mm}$. The donor DM and endothelium were gently stripped off with a dry cellulose sponge or forceps. The donor cornea was initially fixed with 4 cardinal 10-0 nylon sutures (Sharpoint, Angiotech Pharmaceuticals, Inc. Vancouver, Canada) at the $3,6,9$, and 12 o'clock positions. Three different suturing techniques were employed based on the surgeon's preference. These consisted of 16 interrupted sutures, a single running suture with 16 to 18 bites, or a combined technique ( 8 interrupted sutures accompanied by a single 16-bite running suture). The suture bites encompassed approximately $90 \%$ of the thickness of the recipient and donor tissues in all of the suturing techniques. Intraoperative keratoscopy was performed to adjust suture tension. At the conclusion of surgery, dexamethasone $4 \mathrm{mg}$ was injected subconjunctivally. Patients received topical moxifloxacin 0.5\% drops every 6 hours for 30 days and topical prednisolone I\% every 6 hours tapered over 2 to 3 months. If indicated, sodium chloride $5 \%$ drops were prescribed to reduce graft edema and filamentary keratitis, and topical lubricants were administered to hasten epithelial healing. In intractable cases, other interventions, such as bandage contact lens fitting (OmniFlex; Hydron International Co., Ltd., Shanghai, China) or amniotic membrane transplantation were used to treat non-healing epithelial defects. Postoperatively, patients were examined on the first day after surgery and every alternating day postoperatively to assess the status of corneal epithelial healing. Follow-up examinations were scheduled I, 3, 6, 12, 24, 36, 48 , and 60 months postoperatively, with an appointment at least once every 3 months until complete suture removal, and 6 months thereafter. BSCVA, corneal topography, biomicroscopic slit lamp examination, Goldman applanation tonometry, and fundoscopy were performed at each visit. An automated phoropter (CV-5000; Topcon Corp., Tokyo, Japan) and a back-illuminated 19" LED LCD monitor chart (CC-100 XP; Topcon Corp., Tokyo, Japan) were used for visual acuity examinations. Visual acuity was converted to 
logMAR for statistical analysis. BSCVA, $\mathrm{KI}$, and $\mathrm{K} 2$ readings, as well as intra- or postoperative complications or secondary interventions (such as resuturing) were recorded and evaluated. When more than I procedure was required (such as resuturing), the final results were considered for analysis.

The mean and SD were used for descriptive statistics of variables with normal distribution. The Kolmogorov-Smirnov test was used to test the normality of the datasets. A dependent sample t-test was used in the statistical analysis of the comparisons of preoperative and postoperative repeated measures. The level of statistical significance was set at $p<0.05$. Statistical analysis was performed using IBM SPSS Statistics for Windows, Version 20.0. (IBM Corp, Armonk, NY, USA).

\section{Results}

Fifty-seven eyes of 57 (40 male) patients with moderate to advanced keratoconus underwent surgery. The mean age of

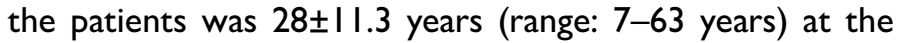
time of the DALK procedure, and were followed for a mean of 48.84 \pm I8.12 months (range: 12-60 months) (Table I).

Table I. Patient characteristics and preoperative data of deep anterior lamellar keratoplasty surgery patients

Age (mean $\pm S D)$, years

$28 \pm 11.3$ (range 7-63)

Follow-up (mean $\pm S D$ ), months

$48.84 \pm 18.12$ (range 12-60)

Male/female

$40 / 17$

Right/left eye

$30 / 27$
The recipient trephination size was 7.25 to $8.0 \mathrm{~mm}$.

The sutures were removed between the 6th and 12th postoperative month. The final outcome analysis was performed after complete suture removal.

The mean BSCVA increased from $I .4 I \pm 0.44 \log M A R$ to $0.87 \pm 0.37 \log M A R$ at the final follow-up $(p<0.00 I)$ (Fig. I). The mean preoperative $\mathrm{KI}$ and $\mathrm{K} 2$ value was $53.5 \pm 8.8$ diopters $(\mathrm{D})$ and $60.6 \pm 8.5 \mathrm{D}$, respectively, while the postoperative $\mathrm{KI}$ and $\mathrm{K} 2$ value was $42.8 \pm \mathrm{I} .2 \mathrm{D}$ and $46.06 \pm \mathrm{I} .3$ D (Fig. 2).

Intraoperatively, microperforations in the DM occurred in 6 eyes (10.5\%). Postoperatively, 8 eyes (14\%) required resuturing, and I patient $(0.2 \%)$ underwent amniotic membrane transplantation (Table 2).

\section{Discussion}

Keratoconus is the most common indication for PK in some countries (6). Several advantages and disadvantages have been published for DALK in comparison with PK $(2,7)$. Some studies $(8,9)$ have published comparable visual outcomes following DALK and PK, while others $(2,10)$ have reported superior visual outcomes after PK. This difference can be attributed to irregularity at the host-donor interface in DALK. DM perforation and conversion to PK have been reported at a rate of $4 \%$ to $39.2 \%$ and $0 \%$ to $14 \%$, respectively (II). The difficult learning curve for the surgical technique may also contribute to differences in success rates of big-bubble formation seen in various studies. We decided to include only successful big-bubble formation cases in this study.

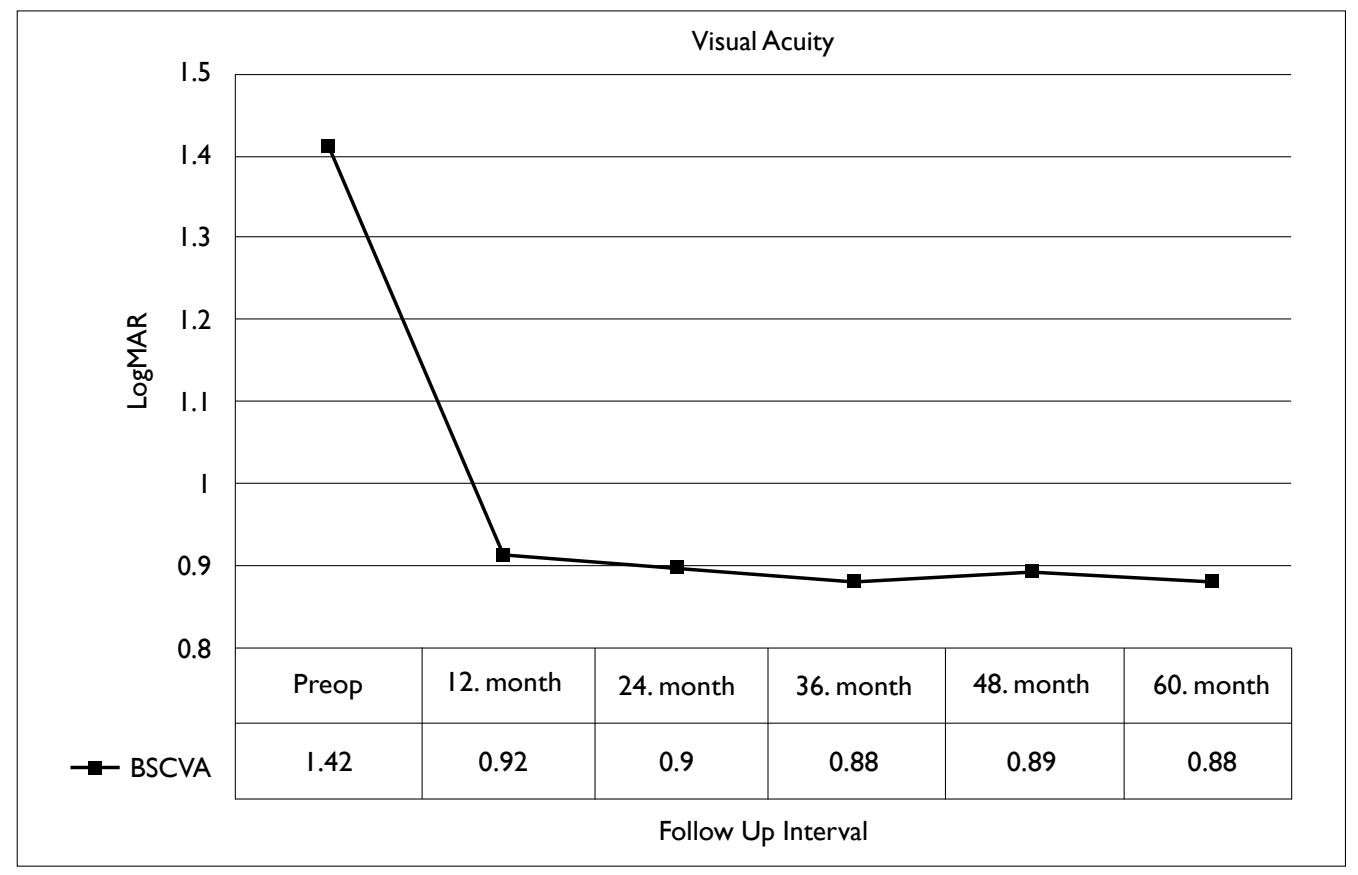

Figure I.Visual acuity over the entire follow-up period.

BSCVA: Best spectacle-corrected visual acuity. 


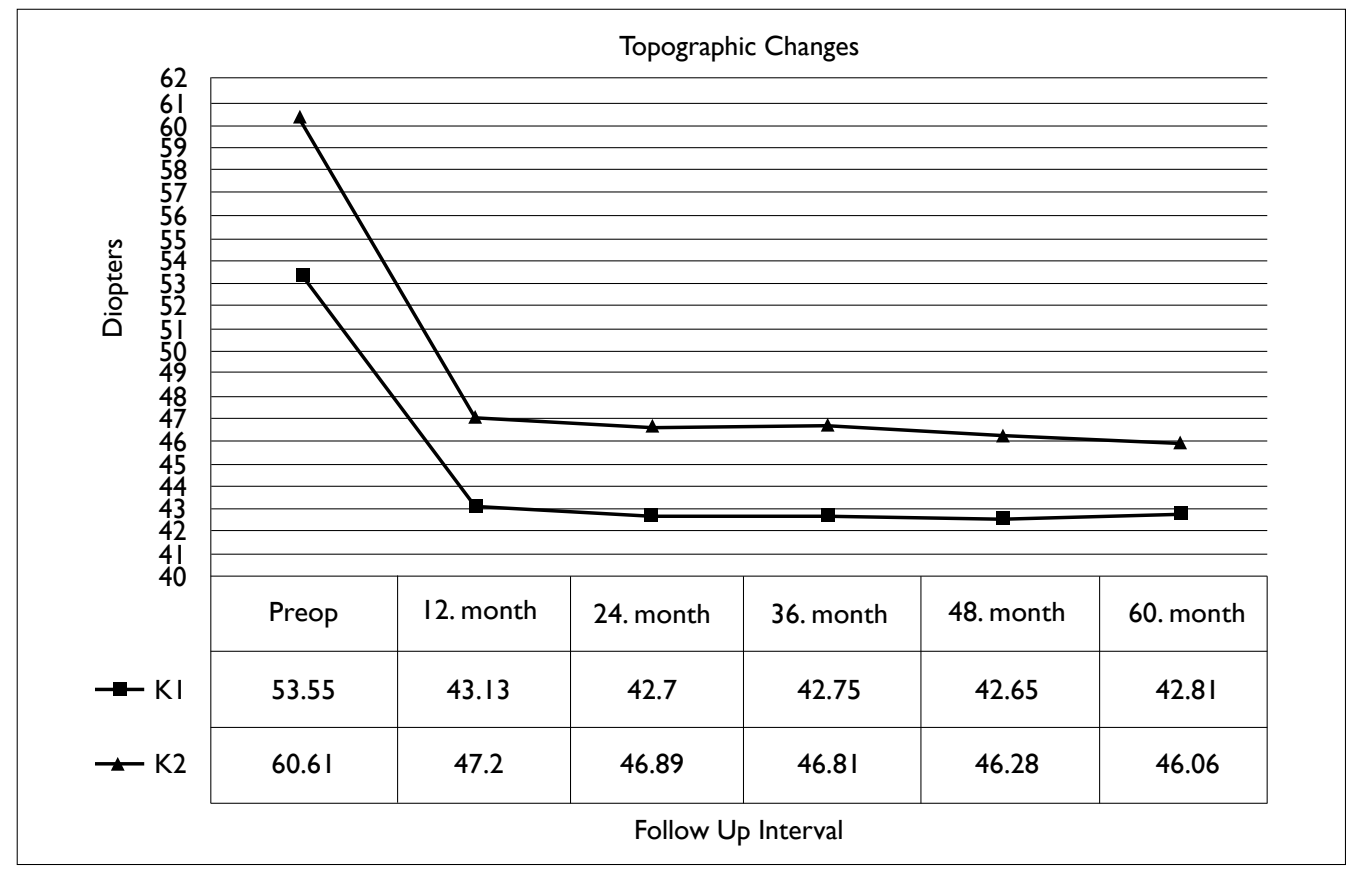

Figure 2. Topographic changes.

Table 2. Complications of deep anterior lamellar keratoplasty

\begin{tabular}{lcc} 
Complication & n & $\%$ \\
\hline Microperforation & 6 & 10.5 \\
Loose suture & 14 & 24.5 \\
Resuturation & 8 & 14 \\
Persistent epithelial defect & $\mathrm{I}$ & 1.7 \\
Amniotic membrane transplantation & $\mathrm{I}$ & 1.7 \\
\hline
\end{tabular}

Although DALK rules out the risk of endothelial rejection, other types of graft rejection (subepithelial and stromal) may still spring up. The clinical features of subepithelial and stromal graft rejections after DALK are very similar to those following PK (I2). In this study, there was no rejection; which may be explained by excluding eyes with a history of VKC, and having too few patients to see rejections.

In our study, the mean BSCVA increased from I.4I \pm 0.44 $\log M A R$ to $0.87 \pm 0.37 \log M A R$ at the final follow-up $(p<0.00 I)$. Romano et al. (I3) observed a corrected distance visual acuity (CDVA) of 20/25 $(0.09 \pm 0.1$ logMAR) at the last control examination after DALK surgery in 158 eyes/I50 consecutive patients with keratoconus with a postoperative follow-up time equal to or greater than 4 years when the CDVA was $20 / 50(0.7 \pm 0.2 \log M A R)$ at the beginning. The length of the follow-up was longer in that study than in ours (76.9 \pm 23.2 months [range: $48-120$ months]) and the study included a larger number of patients.

Javadi et al. (14) found a mean preoperative BSCVA of I.23 $\pm 0.4 \log M A R$ (range: $0.0-2.0 \log M A R$ ), equivalent to
20/400, which improved to $0.26 \pm 0.2$ logMAR (range 0.0 I.4 $\log M A R$ ), equivalent to $20 / 40$, at the final follow-up $(p<0.00 \mathrm{I})$. In our study, the preoperative visual acuity and the BSCVA at the last follow up were lower, but there was a statistically significant increase in visual acuity change.

In this study, the mean preoperative KI keratometry was $53.55 \pm 8.85 \mathrm{D}$ in the flattest meridian, whereas it was $42.81 \pm 1.29 \mathrm{D}$ at the last follow-up. The mean preoperative $\mathrm{K} 2$ keratometry measurement at the steepest meridian was $60.6 I \pm 8.5 \mathrm{I} \mathrm{D}$ and $46.06 \pm 1.3 \mathrm{I} \mathrm{D}$ at the last control examination. The changes in $\mathrm{KI}$ and $\mathrm{K} 2$ values were statistically significant. $(p<0.05)$ In 77 eyes, Javadi et al. (I4) reported a mean preoperative keratometry measurement of $55.5 \mathrm{I} \pm 5.33 \mathrm{D}$ (range: $44.25-7 \mathrm{I} .5 \mathrm{D}$ ) and a postoperative value of $47.04 \pm 2.27 \mathrm{D}$ (range: $42.25-55.5 \mathrm{D})(\mathrm{p}<0.00 \mathrm{I})$.

Behesht-Nejad et al. (I5) performed DALK using the bigbubble technique in 17 eyes with a mean preoperative keratometry of $60.1 \pm 7.3 \mathrm{D}$ and observed a result of $44.86 \pm 2.4$ D 9 months after the procedure $(p<0.0001)$. Although our follow-up period was longer and our research included 57 eyes, the outcomes were similar, particularly the $\mathrm{K} 2$ readings.

The complications experienced are provided in Table 2. A total of 6 eyes $(10.5 \%)$ had a microperforation in the DM, which occurred during air injection or removal of residual corneal stroma, though it was still possible to continue the DALK procedure because the defect was small. Several studies have reported DM perforation during DALK, ranging from $4 \%$ to $39.2 \%$. Microperforation rates are highest with manual dissection (26.3\%) and lowest with the Anwar bigbubble technique $(5.48 \%)$. This variation likely reflects differences in surgeon experience, indications for keratoplasty, 
and surgical technique (II).

In the Javadi et al. (14) study, perforation in the DM occurred in 5 eyes during air injection or removal of residual corneal stroma, and 3 required conversion to PK. Zhang et al. (16) performed DALK in 75 eyes and observed microperforation of the DM in 7 eyes $(9.3 \%)$ and a large tear in the DM in I eye (I.3\%).

In our study, loose sutures were noted in 14 patients $(24.5 \%)$ over the follow-up period; however, only 8 cases required resuturing. This rate was similar to that seen in the Behesht-Nejad (I5) study. A persistent epithelial defect was noted in I patient (I.7\%) and was successfully managed with amniotic membrane transplantation. Reepithelialization was completed at 2 weeks, with some subepithelial haze present. There was no instance of subepithelial or stromal graft rejection, interface wrinkling, vascularization or opacification, fixed dilated pupil, suture abscess, or suture tract vascularization as a complication.

Abdel Hakeem et al. (I7) compared the big-bubble technique with manual dissection in DALK and found that in the big-bubble group, the mean BCVA was significantly better $(p=0.006)$, whereas the mean residual stroma was significantly lower $(p=0.000 \mathrm{I})$ and the interface haze was significantly less $(p=0.018)$ than that found in the predescematic DALK group.

In another study, Keane et al. (18) compared DALK with PK and found that no evidence to support a difference in outcome in the treatment of keratoconus with regard to BCVA at 3 months post-graft or at any of the other time points analyzed (GRADE rating: very low). They also found no evidence of a difference in outcomes with respect to graft survival, final UCVA, or keratometric outcome. They found some evidence that rejection was more likely to occur following PK than DALK (GRADE rating: moderate).

The principal limitations of our study are the retrospective design and the fact that it does not include a comparison with other keratoplasty techniques.

In conclusion, DALK is an appropriate alternative to PK in patients with keratoconus. It eliminates the risk of endothelial graft rejection, preserves globe integrity, and provides acceptable visual function. More extensive studies with a longer follow-up period are required to better understand all of the advantages and disadvantages of DALK.

\section{Disclosures}

Financial Disclosure: This retrospective study was not supported by any company. None of the authors has financial or proprietary interests in any material or method mentioned. These data have not been previously published.

Peer-review: Externally peer-reviewed.

Conflict of Interest: None declared.

Authorship Contributions: Involved in design and conduct of the study (NKB, AA, SA); preparation and review of the study (AA, NKB, MT); data collection (SA, NKB); and statistical analysis (NKB).

\section{References}

I. Arnalich-Montiel F, Alió Del Barrio JL, Alió JL. Corneal surgery in keratoconus: which type, which technique, which outcomes? Eye Vis (Lond) 2016;3:2. [CrossRef]

2. Watson SL, Ramsay A, Dart JK, Bunce C, Craig E. Comparison of deep lamellar keratoplasty and penetrating keratoplasty in patients with keratoconus. Ophthalmology 2004; I I I: |676-82.

3. Shimmura S, Tsubota K. Deep anterior lamellar keratoplasty. Curr Opin Ophthalmol 2006; 17:349-55. [CrossRef]

4. Benson WH, Goosey CB, Prager TC, Goosey JD. Visual improvement as a function of time after lamellar keratoplasty for keratoconus. Am J Ophthalmol 1993; I 6:207-2 I I. [CrossRef]

5. Manche EE, Holland GN, Maloney RK. Deep lamellar keratoplasty using viscoelastic dissection. Arch Ophthalmol 1999; | 17:| 56|-5. [CrossRef]

6. Edwards M, Clover GM, Brookes N, Pendergrast D, Chaulk J, McGhee CN. Indications for corneal transplantation in New Zealand: 1991-1999. Cornea 2002;21:152-5. [CrossRef]

7. Terry MA. The evolution of lamellar grafting techniques over twenty-five years. Cornea 2000;19:6II-6. [CrossRef]

8. Coombes AG, Kirwan JF, Rostron CK. Deep lamellar keratoplasty with lyophilised tissue in the management of keratoconus. Br J Ophthalmol 200 I;85:788-9I. [CrossRef]

9. Sugita J, Kondo J. Deep lamellar keratoplasty with complete removal of pathological stroma for vision improvement. $\mathrm{Br}$ J Ophthalmol 1997;81:184-8. [CrossRef]

10. Funnell CL, Ball J, Noble BA. Comparative cohort study of the outcomes of deep lamellar keratoplasty and penetrating keratoplasty for keratoconus. Eye 2006;20:527-32. [CrossRef]

I I. Karimian F, Feizi S. Deep Anterior Lamellar Keratoplasty: Indications, Surgical Techniques and Complications. Middle East Afr J Ophthalmol 2010;17:28-37.

12. Watson SL, Tuft SJ, Dart JK. Patterns of rejection after deep lamellar keratoplasty. Ophthalmology 2006; I I 3:556-60.

I3. Romano V, lovieno A, Parente G, Soldani AM, Fontana L. Longterm clinical outcomes of deep anterior lamellar keratoplasty in patients with keratoconus. Am J Ophthalmol 20I5; I59:505-II.

14. Javadi MA, Feizi S, Jamali H, Mirbabaee F. Deep Anterior Lamellar Keratoplasty Using the Big-Bubble Technique in Keratoconus. J Ophthalmic Vis Res 2009;4:8-13.

15. Behesht-Nejad AH, Alimardani A, Amoozadeh J, Hashemi $H$. Deep Anterior Lamellar Keratoplasty Using the Big-bubble Technique. Iranian Journal of Ophthalmology 2009;21:15-22.

16. Zhang YM, Wu SQ, Yao YF. Long-term comparison of full-bed deep anterior lamellar keratoplasty and penetrating keratoplasty in treating keratoconus. J Zhejiang Univ Sci B 20I3; 14:438-50.

17. Abdel Hakeem AS, Katamish TA, Khalil DH, Alaaeldin HA. Comparative study between big-bubble technique and manual dissection in deep anterior lamellar keratoplasty. J Egypt Ophthalmol Soc 2016;109:93-7. [CrossRef]

18. Keane M, Coster D, Ziaei M, Williams K. Deep anterior lamellar keratoplasty versus penetrating keratoplasty for treating keratoconus. Cochrane Database Syst Rev 2014:CD009700. 